\title{
Similarity judgments of patterns and maps
}

\author{
DIANE F. HALPERN, HAROLD D. FISHBEIN, and JOEL S. WARM \\ University of Cincinnati, Cincinnati, Ohio 45221
}

\begin{abstract}
An algorithmic technique is described for generating from a common prototype three populations of forms (dots, open forms, and polygons) having different configurational properties. Within each population, subjects rated the similarity between standard stimuli and 20 transformations of these stimuli. The stimuli were represented to the subjects as abstract patterns or as maps. High rank-order correlations were obtained between the ratings given to the various transformations in each form population and in the pattern and map conditions. These correlations led to two conclusions: (1) there was ecological validity in the stimulus factors that influenced similarity judgments in the three form populations, and (2) subjects used analogous processes when making similarity judgments in tasks focusing upon pattern perception and spatial reference systems.
\end{abstract}

Attempts to develop a psychophysics of form have sought to determine the informational parameters used by observers in their two-dimensional spatial organizations of the perceptual world (Dember \& Warm, 1979). Typically, this line of investigation has used a variety of abstract algorithmic patterns generated according to formal construction rules. With stimuli of this sort, the psychophysical approach has identified a number of physical dimensions that play important roles in several aspects of the perception of form. Among these is the perceived similarity of shapes. Judgments of similarity have been found to depend upon configurational properties, such as the compactness, jaggedness, and elongation of the stimuli, and upon transpositional parameters involving rotation and reflection (Zusne, 1970).

While the psychophysical approach has produced insights into the perceived similarity of patterns, it faces a problem centering about the issue of ecological validity. This issue concerns the degree to which the results obtained with one population of algorithmic forms are representative of those to be obtained with other populations of these forms or "real-world" objects (Brunswik, 1956). A careful consideration of this problem led Zusne (1970) to conclude that it is difficult to make direct comparisons of experimental findings across form populations, and, indeed, few systematic efforts have been made to do so. The present investigation was designed to attack the problem of representativeness in the perceived similarity of patterns. Our strategy was to compare the rated similarity of transformed stimuli to standards in three populations of algorithmic forms.

The perception of form is an aspect of research in spatial organization in which the major focus of interest

Requests for reprints should be sent to Diane F. Halpern, Department of Psychology, University of Cincinnati, Cincinnati, Ohio 45221. is upon "how things appear." Another aspect of research involving spatial organization deals with the problem of spatial reference systems or the issue of "where things are" (Pufall \& Shaw, 1973). Recently, this line of investigation has begun to use patterns of a more symbolic sort-maps-to study the information employed in making judgments of spatial position. Such an approach lends itself to a psychophysical analysis similar to that used when the experimental question focuses upon "how things appear." For example, Fehr and Fishbein (Note 1) have found that configurational properties such as axial location and displacement of map elements account for much of the variation in similarity judgments of discrete element dot maps, that is, maps in which dots reflect the spatial positions of buildings or other objects.

Although studies concerning "how things appear" and "where things are" originate from different conceptual frameworks, they frequently employ similar stimuli and analogous psychophysical manipulations. From the broad issue of generality, it is of interest to determine if there is any correspondence in the factors that influence performance when subjects are asked to operate within these two frameworks. Accordingly, a second aspect of this study was to compare the similarity ratings of patterns when the stimuli were represented to subjects as abstract forms or as maps.

\section{METHOD}

\section{Subjects}

Thirty-six students, 18 females and an equal number of males, from the University of Cincinnati served as subjects in order to fulfill a course requirement. None of the students had prior experience with experiments on form perception or the judgment of spatial relations.

\section{Stimuli}

Three form populations consisting of dot, open-form, and polygon patterns were used. A "cell-matching" technique was devised to resolve a difficulty inherent in any attempt to 
compare similarity judgments in different populations of forms. That is, the problem of selecting stimuli that, although obviously different in configurational properties, are nevertheless similar in ways that permit meaningful comparisons.

To generate stimuli, a prototype was first determined by selecting 7 cells at random from an 8 by 8 matrix. Dot figures were constructed from the prototype by placing dots in the cells so selected. Open forms and polygons were constructed from the prototype by connecting the dot elements according to specified rules. For open forms, these rules were: (1) every dot must be connected to another dot with a straight line, (2) lines may cross, and (3) each figure must be open (i.e., not bounded by a closed perimeter). The rules for constructing polygons were: (1) Rule 1 for open forms, (2) lines may not cross, and (3) each figure must be bounded by a closed perimeter.

Three prototypes were generated to produce three standard patterns to be displayed as dot figures, open forms, and polygons. For each standard, 20 transformations (Ts) were constructed to provide several comparison stimuli that varied in similarity to the standard. The Ts were: T1, standard unchanged; $\mathrm{T} 2$ and $\mathrm{T} 3$, alterations in spatial location [the comparison figure was moved to the lower left corner of the field in which it appeared (T2) or to the upper right corner of the field (T3)]; T4 and T5, size modifications [the comparison figure was enlarged by a factor of 1.5 (T4) or reduced by a factor of .5 (T5)]; T6-T10, modifications of spatial orientation (the comparison figure was presented in 90-, 180-, 270-, 225-, or 45-deg rotation, respectively, with respect to the standard); $\mathrm{T} 11$ and $\mathrm{T} 12$, reflection of the standard [right-left reflection (T11), up-down reflection (T12)]; T13 and T14, width-height modification [the width-to-height ratio of this comparison was changed to $1: .5$ (T13) and $.5: .75$ (T14)]; T15 and T16, structural change [in constructing the comparison figure, the upper right dot in the prototype matrix was moved two units left and one unit down (T15), the lower left dot in the prototype matrix was moved two units right and one unit up (T16)]; T17-T20, multiple modifications $(\mathrm{T} 17=\mathrm{T} 2+\mathrm{T} 6$; $\mathrm{T} 18=\mathrm{T} 2+\mathrm{T} 6+\mathrm{T} 12 ; \mathrm{T} 19=\mathrm{T} 4+\mathrm{T} 15 ; \mathrm{T} 20=\mathrm{T} 4+\mathrm{T} 15+\mathrm{T} 16)$. Examples of "cell-matched" dot, open-form, and polygon stimuli and selected Ts are presented in Figure 1.

\section{Apparatus and Procedure}

Subjects were tested individually in an Industrial Acoustics sound chamber. A set of 20 slides containing a standard figure

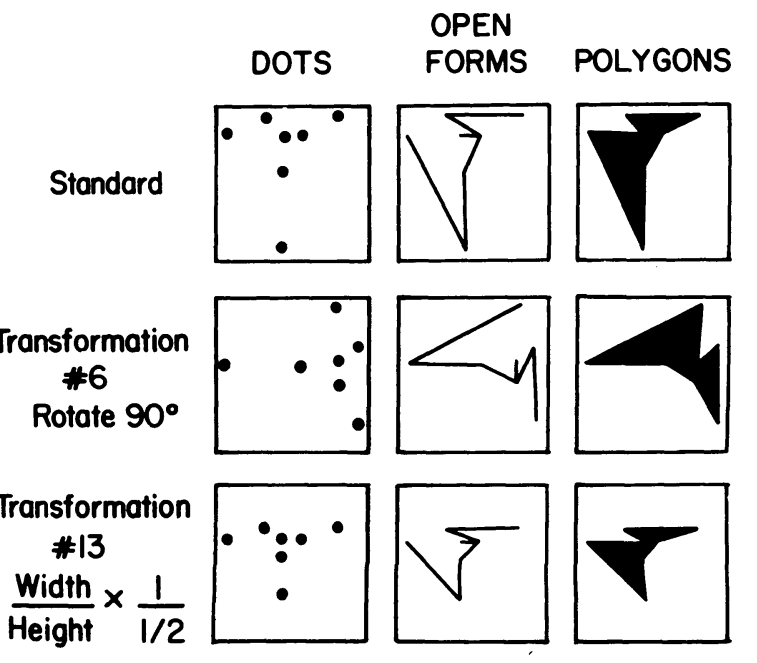

Figure 1. A sample of "cell-matched" stimuli and selected transformations. and one of its Ts was prepared for each of the nine combinations of three standards and three figure types. The stimuli were rear-projected onto a viewing window located at eye level in the subject's chamber. On each stimulus presentation, the subject saw a bipartate field divided by a black line. The standard was centered within the right half of the field and the comparison (transformed) figure appeared in the left half. The comparison was centered within the left half of the field, except in the cases of Ts $2,3,17$, and 18 . For these Ts, the spatial location of the comparison stimulus was that described above. All stimuli appeared as black figures on a white background.

Eighteen subjects were assigned at random (with the restraint of equation for gender) to pattern or map instructional conditions. In the former, the stimuli were described as dot, open-form, or polygon patterns. In the latter, subjects were told that the dot configuration reflected a spatial representation of downtown buildings, the open forms were described as street maps, and the polygons were represented as city boundary or outline maps. Within the two instructional conditions, each subject experienced each of the nine standard/figure-type combinations as he or she progressed through the experiment. The order in which these combinations were experienced was determined by means of a 9 by 9 Latin square. Within each standard/figure-type combination, subjects were exposed to the appropriate set of 20 slides. For each slide, they judged the similarity of the comparison stimulus to its standard. The order of presentation of the slides within a set was varied at random for each subject.

Under all experimental conditions, similarity judgments were obtained in two phases. First, all 20 slides of a given set were presented for familiarization. Exposure time was $2 \mathrm{sec} / \mathrm{slide}$ with a .7-sec interslide interval. During this phase, the subjects were instructed to observe the slides and become acquainted with the range of comparison forms in a set. The set was then represented with the same interslide interval, but with exposure time increased to $8.0 \mathrm{sec}$. During this phase, subjects recorded their judgments in an answer booklet while each slide was exposed. They responded in terms of a 7-category rating scale ranging from "most different from the standard" (1) to "identical to the standard" (7). Subjects were asked to use Category 7 only if the comparison stimulus was identical to the standard in configurational properties and spatial location. Except for this constraint, subjects were free to use any of the remaining categories as they wished.

\section{RESULTS AND DISCUSSION}

Preliminary inspection of the data revealed that similarity ratings for the 20 Ts were essentially equivalent with each of the three standards under all combinations of figure type and pattern/map instructions. Consequently, the data were collapsed across standards. For each subject, median ratings were computed for each $T$ with each of the three figure types.

One aim of this investigation was to compare similarity judgments in different populations of forms. If the stimulus factors that influence similarity judgments in different form populations are ecologically valid, one would expect analogous results in the judgments given to the various Ts among the dot, open-form, and polygon patterns. Means of median similarity ratings for the 20 Ts in each of the three form populations are presented in Figure 2. The figure reveals a remarkable harmony in the distributions of ratings for the $20 \mathrm{Ts}$ 


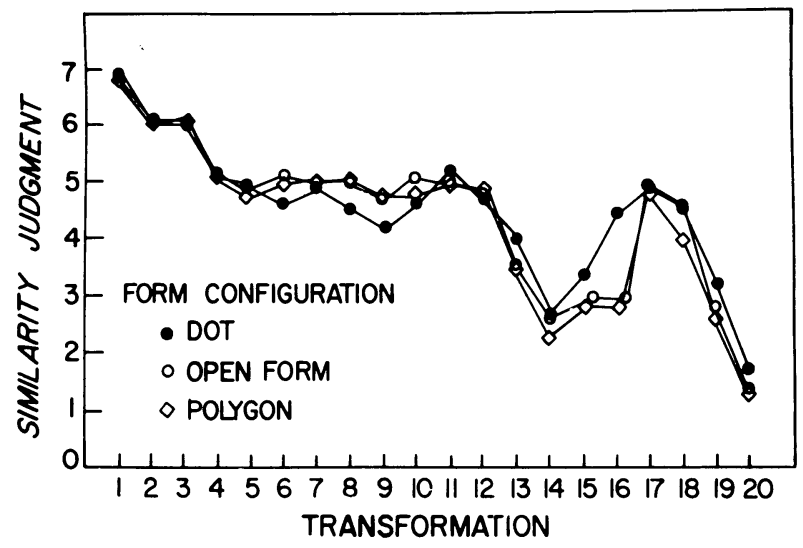

Figure 2. Similarity judgments for various transformations in three form populations. (The transformation scale is nominal.)

in each form population. A numerical index of the strength of these relations is given by Spearman rankorder correlations between the form populations with regard to the ratings given to the various Ts. These values are: dots and open forms, rho $=.90$; dots and polygons, rho $=.85 ;$ open forms and polygons, rho $=.84$ $(\mathrm{p}<.01$ in each case) .

These results indicate that it is indeed possible to make direct comparison of experimental findings across form populations. They also indicate that, with reference to similarity judgments and the types of stimuli used here, the findings obtained with one population of algorithmic forms are representative of those to be obtained with the others. The basis for this representativeness remains to be determined.

Research in pattern perception has stressed a property-list mode of analysis (Dember \& Warm, 1979). According to this approach, patterns are compared in terms of a weighted combination of local features, such as angles and lines, and the presence or absence of particular features is decisive in determining the response given to the patterns in question. The dot patterns, open forms, and polygons used in this investigation share few local features. Therefore, it is difficult to understand how similarity judgments based upon a property-list analysis of the standard and transformed stimuli could produce such closely analogous outcomes in these three populations of forms. On the other hand, recent investigations (Navon, 1977; Tversky, 1977) have pointed to the importance of global structuring in the analysis of form. While the dot, open-form, and polygon figures share few local features, they do have similar global configurations in terms of such attributes as size, axis of symmetry, and elongation (see Figure 1). It is possible that, in the present case, the global characteristics of the standard and transformed stimuli in each population were more salient than the local characteristics in determining perceived similarity, and that this global salience accounts for the harmony in the findings across form populations.
A second aspect of this investigation was to compare the similarity ratings of patterns when the same stimuli are represented to subjects as abstract forms or as maps. Means of median judgments for the 20 Ts under pattern and map instructions are presented in Figure 3. The figure shows a high degree of correspondence among the two instructional conditions in regard to the profiles of similarity ratings for the various Ts. The Spearman rank-order correlation between the ratings given to the various Ts in the pattern and map conditions was .85 $(\mathrm{p}<.01)$.

Evidently, there is more than just methodological parallelism between studies of pattern perception and experiments on spatial reference systems. Subjects seem to employ analogous processes when making similarity judgments of abstract patterns and more symbolic maps. This inference needs to be modified a bit, however. Perusal of Figure 3 reveals that, while there was a general congruence in similarity ratings under pattern and map instructions, a systematic difference between instructional conditions was also evident. In terms of the absolute values of the ratings, map instructions resulted in generally lower scores for Ts $6,7,8,9,10,11,12,17$, and 18 than did pattern instructions. All of these Ts involved rotations or reflections of the standard. Evidence is available to indicate that rotation and reflection manipulations influence perceptual performance in a variety of pattern perception and spatial judgment situations (Howard \& Templeton, 1966; Nigl \& Fishbein, 1974). The present data suggest that these manipulations may be more salient for the perceived similarity of maps than for that of abstract patterns. Such a suggestion has intuitive appeal, because a rotated or reflected map is no longer spatially veridical: It does not correspond to spatial location. Colloquially, it is harder to get from here to there with a rotated or reflected map than with one not subjected to such transformations.

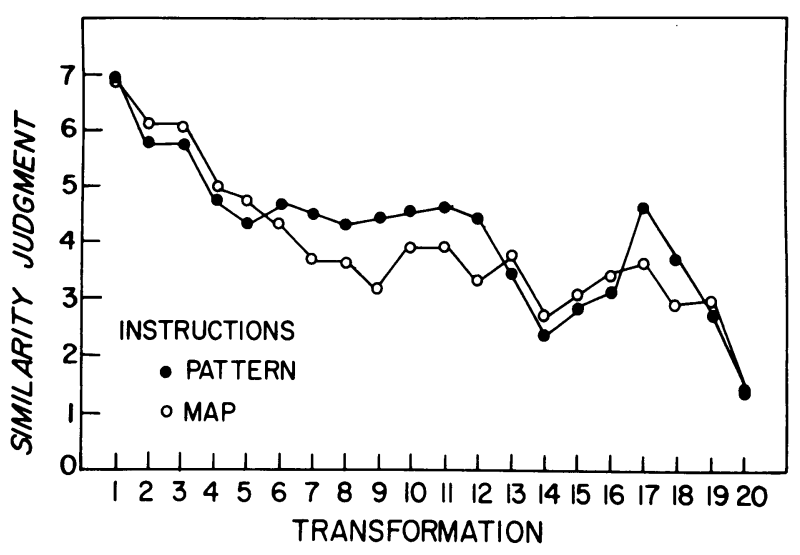

Figure 3. Similarity judgments for various transformations under pattern and map instructions. (The transformation scale is nominal.) 


\section{REFERENCE NOTE}

1. Fehr, L. A., \& Fishbein, H. D. Children's and adult's organization of spatial relations. Unpublished manuscript, University of Cincinnati, 1976.

\section{REFERENCES}

BRUnSWIK, E. Perception and the representative design of psychological experiments. Berkeley: University of California Press, 1956.

Dember, W. N., \& Warm, J. S. Psychology of perception (2nd ed.). New York: Holt, Rinehart, \& Winston, 1979.

How ARD, I. P.. \& TEMPleton, W. B. Human spatial orientation. New York: Wiley, 1966.
Navon, D. Forest before trees: The precedence of global features in visual perception. Cognitive Psychology, 1977, 9. 353-383.

Nigl, A. J., \& Fishbein, H. D. Perception and conception in coordination of perspectives. Developmental Psychology, 1974, $10,858-866$.

Pufall, P. B., \& Shaw, R. E. Analysis of the development of children's spatial reference systems. Cognitive Psychology, 1973, 5, 151-175.

TVersky, A. Features of similarity. Psychological Review, 1977, 84, 327-352.

Zusne, L. Visual perception of form. New York: Academic Press, 1970.

(Received for publication September 20, 1978.) 